AC 2009-821: FLUORESCENCE CORRELATION SPECTROSCOPY WITH CONFOCAL DETECTION

Asad Yousuf, Savannah State University

Yan Zeng, Savannah State University 


\title{
Fluorescence Correlation Spectroscopy \\ with Confocal Detection
}

\begin{abstract}
Fluorescence correlation spectroscopy (FCS) is one of the many different methods of high-resolution spatial and temporal analysis of low concentrated bio-molecules. One outstanding feature of this method is its comparable noninvasiveness, which makes it perfectly suited for measurements inside living cells. Comparing to other fluorescence techniques, the parameter of primary interest is not the emission intensity itself, but spontaneous intensity fluctuations caused by the minute deviations of the small system from thermal equilibrium. Traditional FCS measurements suffered from poor signal-tonoise ratios, mainly because of large ensemble numbers of molecules flowing in and out of the detection area. Researchers managed to reduce the concentrations and observation volumes such that only few molecules are simultaneously detected. The final breakthrough was achieved by Rigler and his coworkers by combining the FCS techniques with confocal detection. The purpose of this paper is to describe the design/development of the FCS setup. The setup is modeled to collect the fluorescence light from the sample and is passed through the emission filter. The pinhole in the image plane blocks any fluorescence light not originating from the focal region. Afterwards, the light is focused onto the detector, preferably a photomultiplier with single photon sensitivity. Future plan is to incorporate this research experience into our Summer Undergraduate Research Training Program (SURTP) for our students.
\end{abstract}

\section{Introduction}

Spectroscopy (1) is originally the study of the interaction between radiation and matter as a function of wavelength. In fact, historically, spectroscopy referred to the use of visible light dispersed according to its wavelength. Later the concept was expanded greatly to comprise any measurement of a quantity as function of either wavelength or frequency. Spectrometry is the spectroscopic technique used to assess the concentration or amount of a given species. In those cases, the instrument that performs such measurements is a spectrometer. Spectroscopy/spectrometry $(2,3,4)$ is often used in physical and analytical chemistry for the identification of substances through the spectrum emitted from or absorbed by them. The science of spectroscopy can be part of the curriculum for chemistry and biochemistry engineering major. The spectroscopy course should include an introduction to light and its properties, the electromagnetic spectrum, types of light both visible and invisible to the human eye, and the effects of light interaction with matter. Also the descriptions of common spectroscopic analysis techniques and applications of spectroscopy should be included.

Fluorescence spectroscopy uses higher energy photons to excite a sample, which will then emit lower energy photons. This technique has become popular for its biochemical and medical applications. Fluorescence correlation spectroscopy (FCS) $(5,6,7)$ is one of the 
many different methods of high-resolution spatial and temporal analysis of low concentrated biomolecules. One outstanding feature of this method is its comparable noninvasiveness, which makes it perfectly suited for measurements inside living cells. Comparing to other fluorescence techniques, the parameter of primary interest is not the emission intensity itself, but spontaneous intensity fluctuations caused by the minute deviations of the small system from thermal equilibrium.

Traditional FCS measurements suffered from poor signal-to-noise ratios, mainly because of large ensemble numbers of molecules flowing in and out of the detection area. Researchers managed to reduce the concentrations and observation volumes such that only few molecules are simultaneously detected. The final breakthrough was achieved by Rigler(6) and his coworkers by combining the FCS technique with confocal detection.

As shown in figure 1, this is the schematic drawing of confocal FCS setup. The incoming laser light is strongly focused by a high numerical aperture objective to a diffraction limited spot. The fluorescence light from the sample is collected by the same objective and passed through the dichotic and the emission filter. The pinhole in the image plane blocks any fluorescence light not originating from the focal region. Afterwards, the light is focused onto the detector, preferably a photomultiplier (PM) with single photon sensitivity.

The confocal FCS method limits the detection volume to less than one femtoliter. Consequently, the signal fluctuations induced by molecules diffusing into or out of the focal volume are large enough to yield good signal-to-noise ratios. Nowadays, it has already become as one of the standard tools with high-resolution and very short data acquisition times (around nano-seconds) for intracellular measurements in biophysics research area.

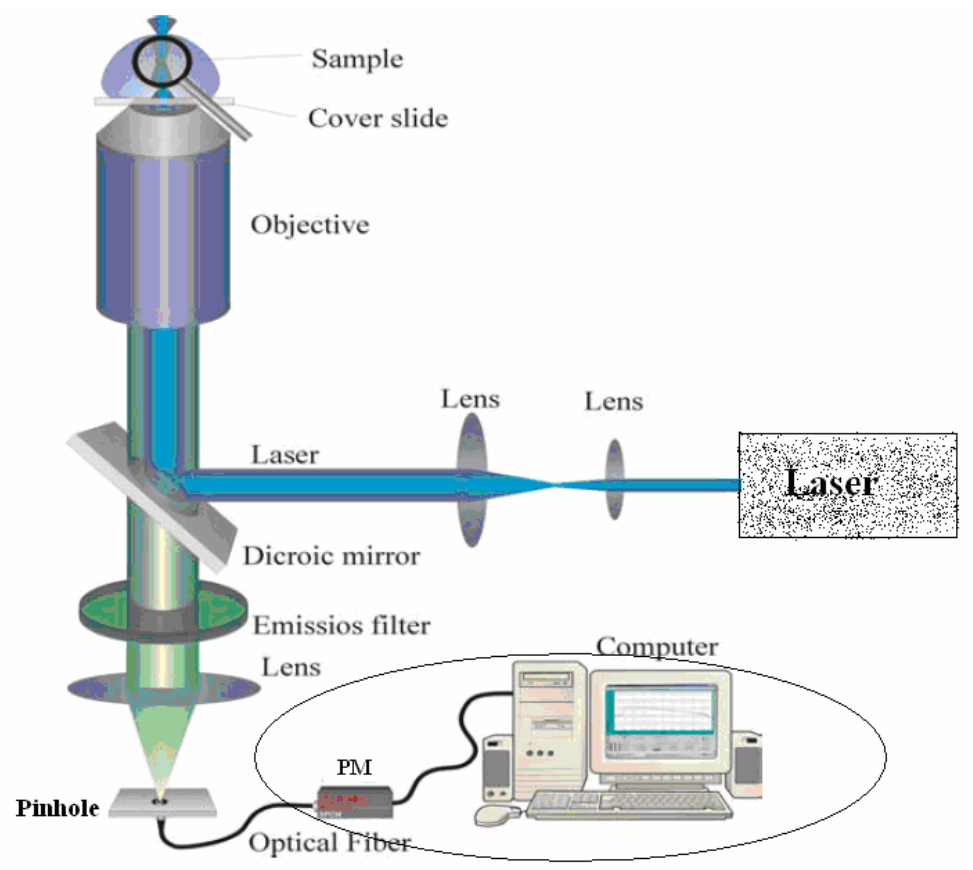

Fig.1: Schematic drawing of confocal FCS setup 


\section{Experiment Setup and Autocorrelation Method}

FCS measurements were conducted on a setup build in our lab, similar to the traditional one. Except in order to reduce the artifacts introduced by the after-pulsing of the detectors, at the back of the pinhole the detection part (as circled in Fig. 1) was replaced by what is shown in Fig. 2. Here the beam is divided in two by a beam splitter (BS) and focused onto two Avalanche photo-counting modules (APD). The signals from these two detectors are fed into a correlator (CORR), and the cross-correlation of the emitted light is collected. The excitation light is provided by an argon laser of 514.5-nm line and the excitation power is kept low at $25 \mathrm{~mW}$ to avoid photon bleaching. The objective lens used is Olympus 60x, numerical aperture 0.9, oil immersion. The excitation light is rejected while passing through the high-pass dichotic mirror and an emission filter. The emitted light is then focused onto a 25 -mm-diameter pinhole.

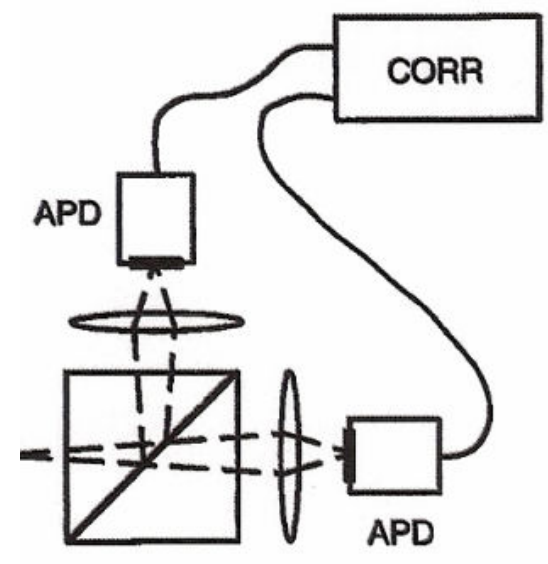

BS

Fig.2: Detection part of our confocal FCS setup

The intensity of fluorescence $I(t)$ emitted by the molecules in the focal spot is recorded photon by photon by the Avalanche photo-counting modules (APD). The correlator (CORR) auto correlates the two signals from APD and output an autocorrelation function $G(t)$. Assuming constant excitation power, the fluctuations of the fluorescence signal are defined as:

$$
\delta I(t)=I(t)-\langle I(t)\rangle
$$

$\operatorname{Here}\langle I(t)\rangle=\frac{1}{T} \int_{0}^{T} I(t) d t$.

If all fluctuations arise only from changes in the local concentration $\delta C$ within the effective volume $V_{\text {eff }}$ of the focal spot, the variation may be written as

$$
\delta I(t)=\int_{V_{\text {eff }}} W(r) \delta(\eta C(r, t)) \cdot d V
$$

Here $W(r)$ describes the spatial distribution of the emitted light and $\eta$ is a measure for the signal-to-noise ratio of the measurement.

The normalized autocorrelation function is defined as: 


$$
G(\tau)=\frac{\langle\delta I(t) \cdot \delta I(t+\tau)\rangle}{\langle I(t)\rangle^{2}}
$$

The signal is analyzed with respect to its self-similarity after the lag time $\tau$. Substituting equation (2) into equation (3) and applying simplification assumptions, we can finally calculate the autocorrelation function for one freely diffusing species of molecules

$$
G(\tau)=\frac{1}{V_{\text {eff }}\langle C\rangle} \cdot \frac{1}{\left(1+\frac{\tau}{\tau_{D}}\right)} \cdot \frac{1}{\sqrt{1+\left(\frac{r_{0}}{z_{0}}\right)^{2} \cdot \frac{\tau}{\tau_{D}}}}
$$

Here dimensions $r_{0}, z_{0}$ can be obtained from calibration measurements, and lateral diffusion time $\tau_{D}$ can be obtained from $r_{0}^{2} / 4 D$ ( $D$ is the diffusion coefficient).

\section{Results and conclusion}

As shown in Fig. 3, here is the interface of the ALV correlator. We obtained Input Count Rate from Avalanche photo-counting modules (APD) and put into ALV correlator (CORR) for Normalized Intensity Correlation Function. Examples of Input Count Rate vs. time graph and Normalized Intensity Correlation Function vs. time graph are shown in Fig. 4 and Fig. 5.

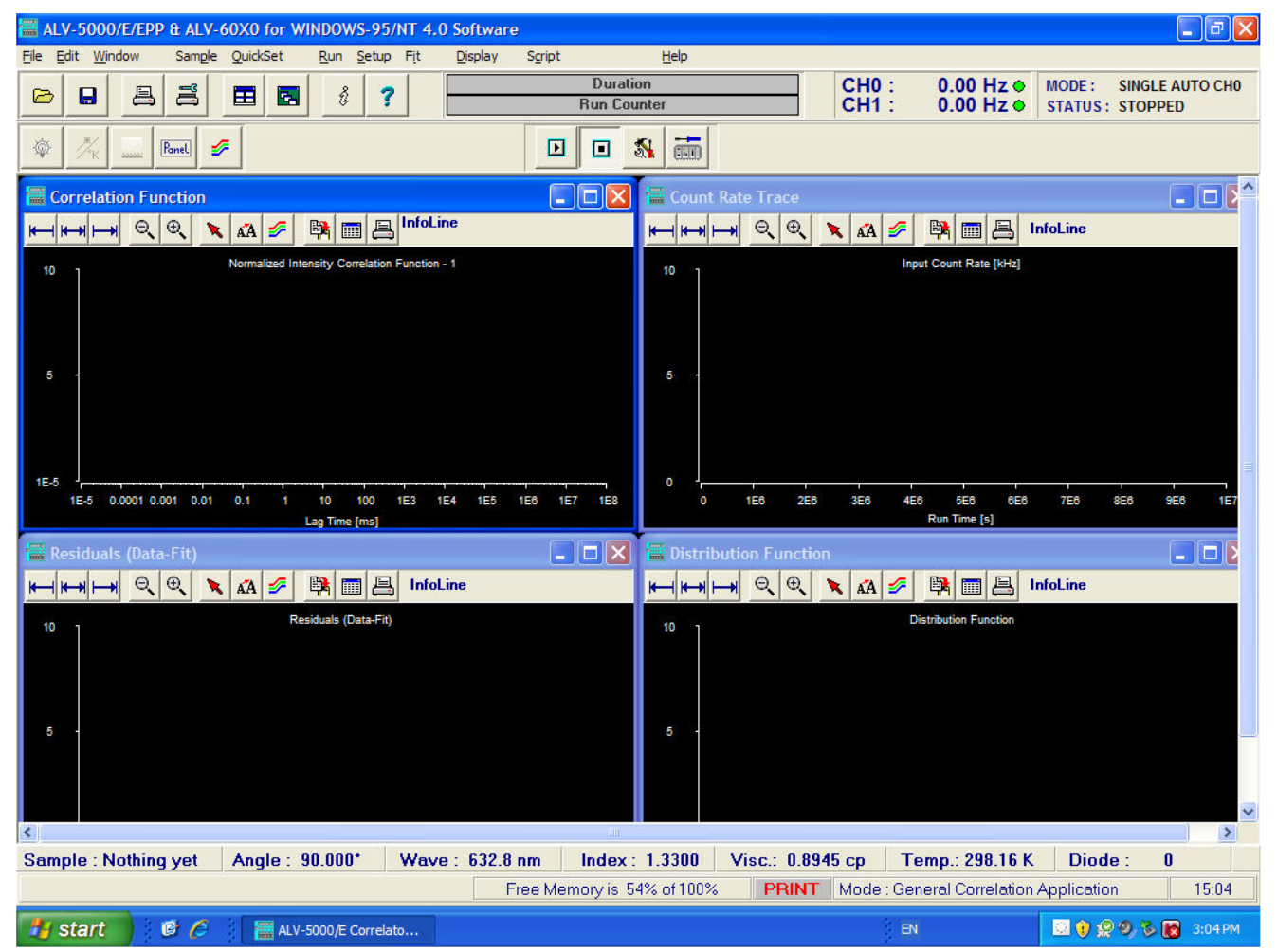

Fig.3: Interface of ALV correlator 


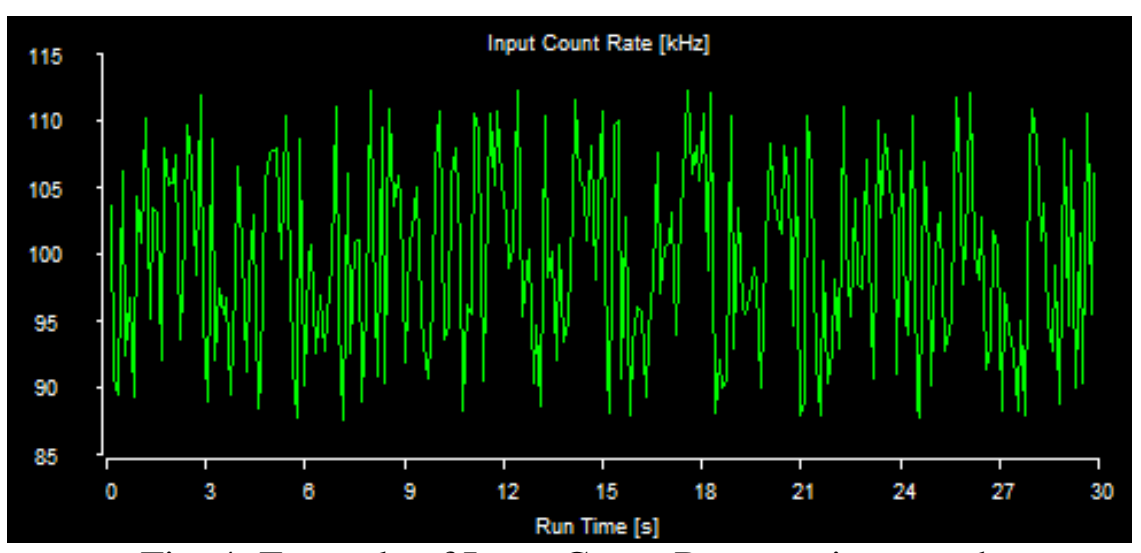

Fig. 4: Example of Input Count Rate vs. time graph

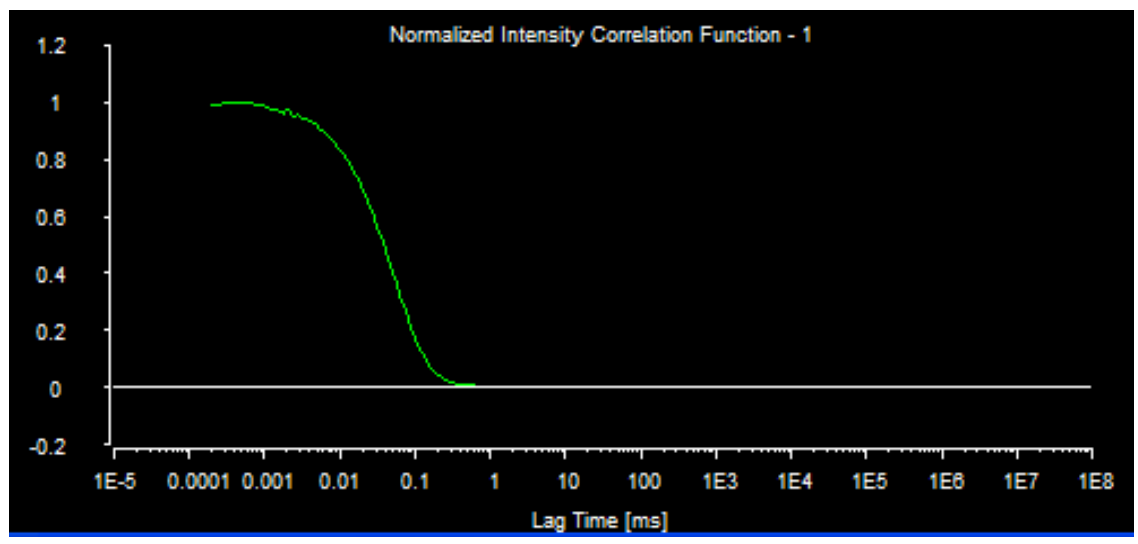

Fig. 5: Example of Normalized Intensity Correlation Function vs. time graph

Applying the FCS with confocal detection method on different chemical structure labeled with fluorescence dyes, people can do lots of measurement on biological samples. Especially in recent year, many publications are based on the FCS with confocal detection method $(8,9,10)$. We believe this would be a good lab exercise for the students and advanced experiment skill for those students who will conduct their research in chemical physics area in the future.

\section{References}

1. "Spectroscopy and Principles of Spectroscopy" Clark, R.N. Manual of Remote Sensing, A. Rencz, Editor, John Wiley and Sons, Inc. 1999.

2. "Reflectance Spectroscopy: Quantitative Analysis Techniques for Remote Sensing Applications", Clark, R.N. and T.L. Roush, J. Geophys. Res., 89, 6329-6340, 1984. 3. "Mapping with Imaging Spectrometer Data Using the Complete Band Shape LeastSquares Algorithm Simultaneously Fit to Multiple Spectral Features from Multiple Materials", Clark, R.N., G.A. Swayze, A. Gallagher, N. Gorelick, and F. Kruse, Proceedings of the Third Airborne Visible/Infrared Imaging Spectrometer (AVIRIS) Workshop, JPL Publication 91-28, 2-3, 1991

4. "Mapping Minerals with Imaging Spectroscopy", Clark, R.N., G.A. Swayze, and A. Gallagher, U.S. Geological Survey, Office of Mineral Resources Bulletin 2039, pp. 141150, 1993. 
5. Mairi, S., Haupts, U.\&Webb, W. W. (1997) Proc. Natl. Acad. Sci.USA 94, 1175311757.

6. Rigler, R., Mets, U“., Widengren, J. \& Kask, P. (1993) Eur.Biophys. J. 22, 169-175.

7. Magde, D., Elson, E. \& Webb, W. W. (1972) Phys. Rev. Lett. 29,705-708.

8. G.Bonnet, O.Krichevsky, and A.Libchaber. (1998) Proc.Natl.Acad.Sci.USA 95, 86028606.

9. G.Bonnet et al. (1999) Proc.Natl.Acad.Sci.USA 96, 6171-6176.

10. G.Bonnet, A.Libchaber, and O.Krichevsky. (2003) Phys.Rev.Lett. 90, 138101. 